\title{
Reconstruction of midface defect from idiopathic destructive process using Medpor implant
}

Joel Franco $^{b}$

Michael S. Harris ${ }^{a}$

Dominic Vernon ${ }^{\mathrm{a}}$

Taha Z. Shipchandler ${ }^{a}$

a. Indiana University School of Medicine, Indianapolis, IN, Department of Otolaryngology - Head \& Neck Surgery, Indiana University School of Medicine, Indianapolis, IN, United States

b. Saint Louis University School of Medicine, Department of Otolaryngology - Head \& Neck Surgery, Saint Louis University School of Medicine, St. Louis, MO, United States

\begin{abstract}
Importance: Reconstruction of the midface remains a challenging task for even the most experienced surgeon, with a host of reconstructive options including free tissue transfer, allografts, or prosthetic implants. Presented here is a case of idiopathic bony destruction of the right midface in a 19 year old female, creating a unique defect requiring repair.
\end{abstract}

Objective: Demonstrate a unique case of severe maxillary degeneration and discuss the associated reconstructive challenges and final repair with a prosthetic implant.

Design: Case report.

Results: The patient presented with a 7 month history of an idiopathic progressive deformity of the right cheek. Computed tomography of the paranasal sinuses revealed extensive bone loss of the right midface

This is the author's manuscript of the article published in final edited form as:

Franco, J., Harris, M. S., Vernon, D., \& Shipchandler, T. Z. (2017). Reconstruction of midface defect from idiopathic destructive process using Medpor implant. American Journal of Otolaryngology, 38(3), 351-353. https://doi.org/10.1016/j.amjoto.2017.01.007 
and orbit. The patient underwent facial reconstruction using a customized Medpor (Stryker Corp, Kalamazoo, MI) implant. At 6 month follow-up the patient and physician were both pleased with the patient's overall appearance. The patient did have some residual lower lid retraction present as well as some lateral pull at the lateral canthus outward from the orbit itself.

Conclusions: Preoperative planning for midface reconstruction requires a deep understanding of the aesthetic, functional, and supportive roles this structure holds. Computer assistance allows the creation of custom made implants, providing the reconstructive surgeon with innovative options for reconstruction with minimal morbidity to the patient. As the technology around the design and creation of the custom implants continues to improve, the role of computer assistance in reconstruction will become more prominent.

\section{Introduction}

The structural, functional, and aesthetic complications of loss of midface support resulting from maxillary defects are uniquely devastating. Structurally and functionally the maxilla serves as the principal separation of the nasal and oral cavities, it constitutes the anterior orbital floor, provides resistance to the forces of mastication, and serves as an anchor to the upper dentition and facial muscles. Aesthetically, this unique bone provides facial contour giving an individual a unique appearance. The etiologies of such defects are varied ranging from trauma to neoplastic causes (1-2).

Currently, there are a number of options available for major maxillary reconstructions. These options include the usage of microvascular free tissue transfer, prosthetic implants, allograft transplants, and tissue engineering. Given this complexity, the approach for reconstruction is often dictated by each patient's unique defect. Some authors advocate for osteomyocutaneous free tissue transfer as the gold standard for large defects. However, not every deficit necessitates such extensive surgery with its associated comorbidities. Unfortunately, no single reconstructive technique accomplishes the goals of 
fully returning the premorbid structural, functional, and aesthetic roles of the maxilla, and it benefits the reconstructive surgeon to seek out the most appropriate method of repair on a case by case basis. (1-4).

This report illustrates a case of idiopathic extensive bone loss of the right midface in a 19-yearold female. The associated reconstruction with a Medpor (Stryker Corp., Kalamazoo, MI) implant and reconstructive challenges are discussed.

\section{Case report}

A 19-year-old female with an unremarkable past medical history and no antecedent facial trauma presented with a depression of the right maxillary eminence progressing over a 7 month period. She reported facial asymmetry arising shortly after a right maxillary dental extraction for severe periodontal disease. Axial high-resolution (0.4 mm slice) non contrast computed tomography of the paranasal sinuses with coronal and sagittal reconstructed images and 3D reconstruction demonstrated extensive bony loss of the right midface including absence of lateral, anterior, and inferior right maxillary sinus walls. Imaging also revealed absence of the right inferior orbital rim from the infraorbital foramen to the zygomatic arch (Figs. 1, 2). She underwent an endoscopic nasal exam with right maxillary sinus biopsy and biopsy of the remnant maxilla at our institution, which was consistent with chronic sinusitis and normal bone.

Initial differential diagnosis for an indolent fibro-osseous destructive processes capable of causing hemifacial atrophy included Wegener granulomatosis, localized scleroderma, and osteolytic syndromes such as Gorham Stout or Perry Rhomberg. Workup with the assistance of rheumatology consultation was ultimately nondiagnostic. A plain film of the chest did not reveal pulmonary nodules and erythrocyte sedimentation rate (ESR), C-reactive protein, anti-nuclear antibody (ANA), rheumatoid factor (RF), cytoplasmic anti-neutrophil cytoplasmic antibodies (cANCA), and perinuclear anti-neutrophil cytoplasmic antibodies (pANCA) were all within normal limits. Biopsy of a minor salivary gland from the right alveolus was significant only for mild chronic inflammation. 
A plan was made to reconstruct and restore facial contour and the integrity of the inferior orbital rim using a custom made Medpor implant.

\subsection{Reconstruction}

A resin model of the patient's facial skeleton was created (Fig. 3). Design of the custom made facial implant is based on high resolution, thin cut, computed tomographic scans with 3-dimensional reconstructions. The level of asymmetry is assessed between the right and left sides of the maxilla. With the unaffected side defining the normal side, a software analysis is performed to create dimensions for a 3-dimensional Medpor implant (Fig. 4). Using this method, Medpor implants can be created in an infinite number of ways to contour to a patient's unique facial skeleton.

\subsection{Surgical technique}

A canthotomy and inferior cantholysis were performed in order to expose the lower lid, and dissection in the preseptal plane was performed. Medially, an inferior orbital rim remnant was identified and dissection was performed in the subperiosteal plane over this remnant.

Laterally, dissection was performed to create a pocket that met up with the zygomatic arch. An incision was made in the sublabial region, where dissection was again performed in the subperiosteal plane medially along the medial vertical buttress and nasomaxillary buttress. The buttresses were noted to be very malformed and underdeveloped. Laterally, there was no bone present. Therefore, a plane deep to the muscles of facial expression and deep to the facial nerve insertions was created and connected with the plane superiorly under the eyelid. Once the plane was connected, a larger pocket was created to accommodate the malar implant. The initial portion of the superior lateral orbital rim reconstructed was along the frontal process, where a $6 \mathrm{~mm}$ bit was used to drill holes through the implant and the frontal process of the zygoma. Screws of $6 \mathrm{~mm}$ were then placed through this area to fixate these portions.

The inferior rim and midface reconstruction was also performed. The implant was connected to the remnant nasomaxillary process and secured in position using four $8 \mathrm{~mm}$ screws. A large portion of the 
implant augmented the malar region. A portion of the implant was placed subperiosteally. This constituted the midface reconstruction.

At 6 month follow up the patient and physician were both pleased with the patient's overall appearance. A degree of residual lower lid retraction was present as well as some lateral pull at the lateral canthus requiring further procedures.

\section{Discussion}

Maxillary defects represent a challenging problem to the facial reconstructive surgeon. In the case of the young patient presented here, an idiopathic process resulted in significant midface deformity with concomitant structural, functional, and aesthetic morbidity. While there currently does not exist a reconstructive option that can return a patient such as this to their premorbid state, reconstructive options include microvascular free tissue transfer (myocutaneous or osteomyocutaneous), prosthetic implants, allograft transplants, and tissue engineering [1-5].

Medpor is a porous polyethylene that is commonly used in craniofacial surgery to correct skeletal defects. Porous polyethylene demonstrates many benefits in reconstructive surgery: 1) it is stable over time, with little evidence demonstrating its degradation; 2) it is porous, allowing bone and soft tissue ingrowth; 3) it obviates donor site morbidity associated with autologous grafts or flaps. Additionally, with the advent of computer-assisted designs, it is possible to have a custom designed implant that closely matches the defect. Finally, Medpor is radiolucent on magnetic resonance imaging (MRI) and CT making it easy examine nearby soft tissue without implant artifact [6].

Although rare, the most commonly reported complications associated with using porous polyethylene implants in reconstructive surgery are persistent pain, paresthesia, implant exposure, infection, and subsequent graft removal [7]. Yaremchuk et al. published a review of 370 porous polyethylene implants in 162 patients. The implants were used in multiple clinical scenarios and implanted in multiple locations. In the 370 implants, there were zero reported cases of extrusion, 
migration, or capsule formation. Yaremchuk did report an 11\% reoperation rate due either to infection (3\%) or displeasing contour (8\%) [8]. In 2005, Cenzi et al. published a review of 285 Medpor implants used in 187 patients. An overall negative outcome rate of 6.31\% was reported, consisting chiefly of implant exposure with subsequent infection [7].

For the case reported here, a good structural, functional, and aesthetic outcome was achieved although a degree of residual lower lid retraction as well as some lateral pull at the lateral canthus was noted, requiring further procedures. Use of a custom made Medpor implant was chosen here given the maxilla defect size, significant structural morbidity, and anticipated aesthetic advantages and desire to obviate donor site morbidity associated with microvascular free tissue transfer. In conclusion, use of porous polyethylene implants are a valuable tool for the facial reconstructive surgeon when faced with large defects of the midface requiring complex contouring.

\section{Conclusion}

Reconstruction of midface defects remains a challenging task, with a multitude of reconstructive options available to the surgeon. The use of custom designed porous polyethylene implants is a viable option for patients with primarily bony defects and minimal soft tissue loss, sparing them the comorbidities associated with microvascular tissue transfer. 


\section{References}

[1] Dalgorf D, Higgins K. Reconstruction of the midface and maxilla. Curr Opin Otolaryngol Head Neck Surg 2008;16(4):303-11.

[2] O'Connell DA, Futran ND. Reconstruction of the midface and maxilla. Curr Opin Otolaryngol Head Neck Surg 2010;18(4):304-10.

[3] Shrime MG, Gilbert RW. Reconstruction of the midface and maxilla. Facial Plast Surg Clin North Am 2009;17(2):211-23.

[4] Costa H, Zenha H, Sequeira, et al. Microsurgical reconstruction of the maxilla: algorithm and concepts. J Plast Reconstr Aesthet Surg 2015;65(5):89-104.

[5] Cho YR, Gosain AK. Biomaterials in craniofacial reconstruction. Clin Plast Surg 2004; 31(3):377-85.

[6] Rudman K, Hoekzema C, Rhee J. Computer-assisted innovations in craniofacial surgery. Facial Plast Surg 2011;27(4):358-65.

[7] Cenzi R, Farina A, Zuccarino L, et al. Clinical outcome of 285 medpor grafts used for craniofacial reconstruction. J Craniofac Surg 2005;16(4):526-30.

[8] Yaremchuk MJ. Facial skeletal reconstruction using porous polyethylene implants. Plast Reconstr Surg 2003;11:1818-27. 
Fig. 1. Computed tomograph (CT) of the paranasal sinuses with coronal reconstructed images in bone windows demonstrating extensive bone loss of the right midface including absence of lateral, anterior, and inferior right maxillary sinus walls.

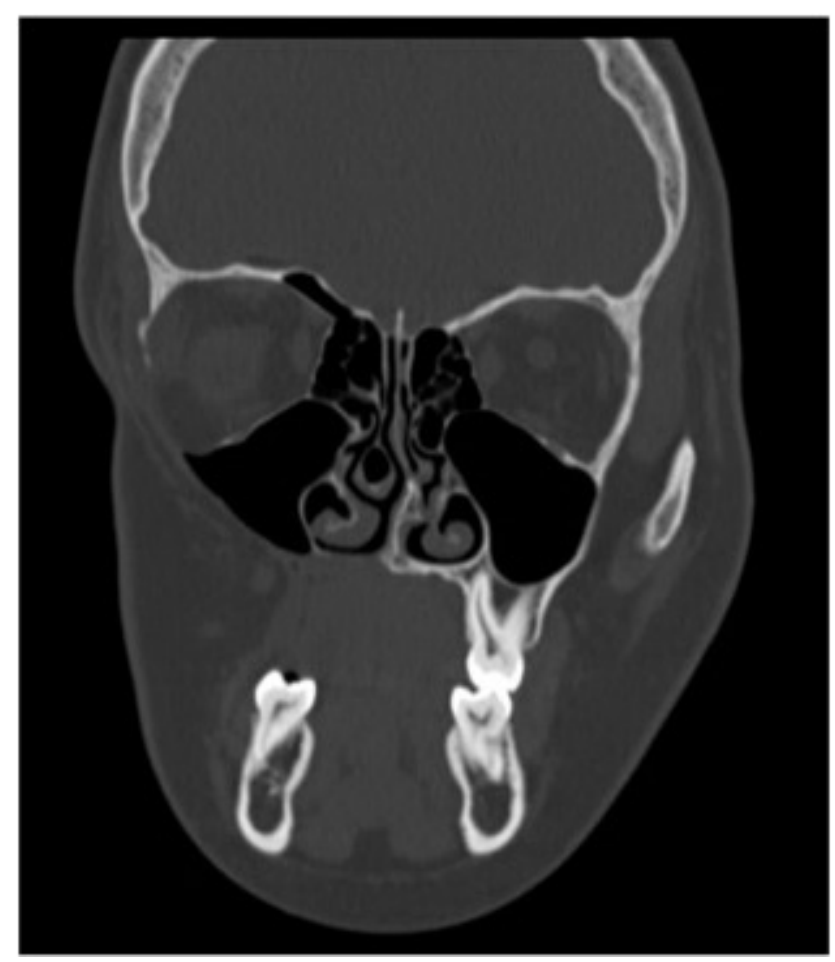

Fig. 2. 3D Reconstruction of noncontrast computed tomograph (CT) of paranasal sinuses. 


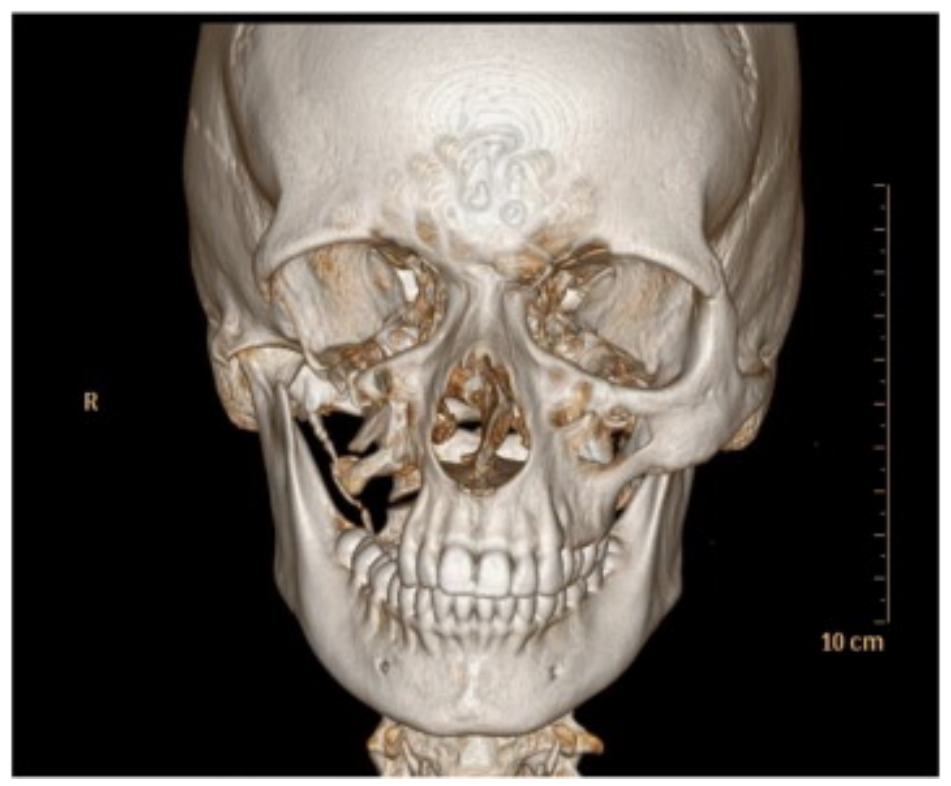

Fig. 3. Resin 3D model of the patient's facial skeleton.

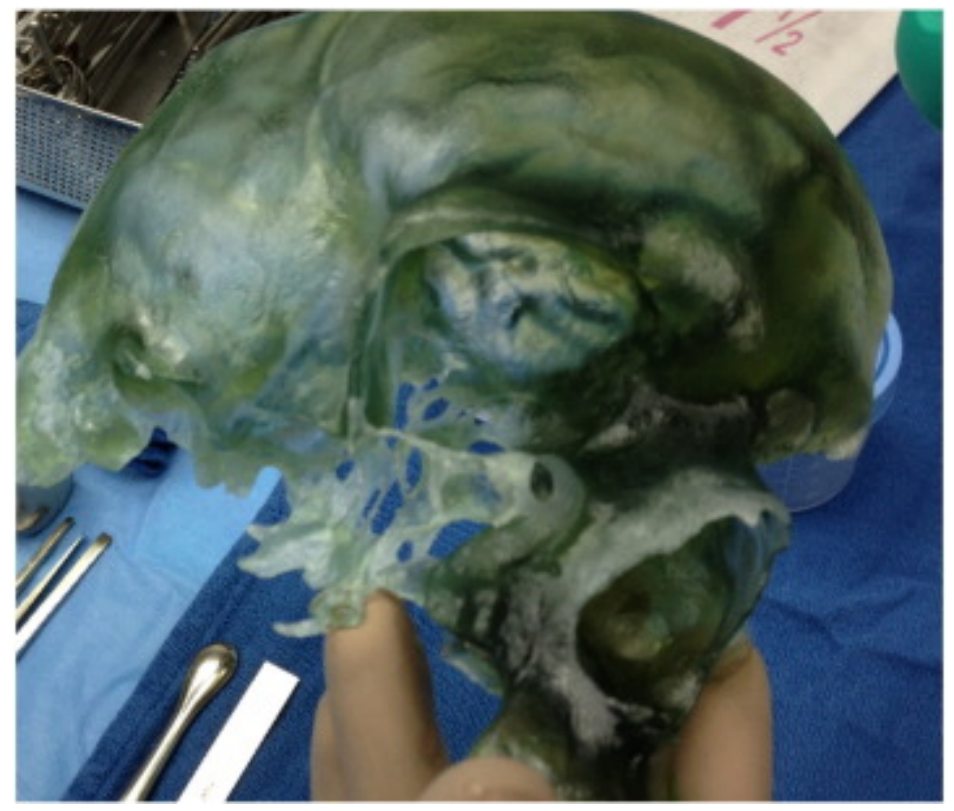

Fig. 4. Planned reconstitution of facial defect using custom-made Medpor implant. 


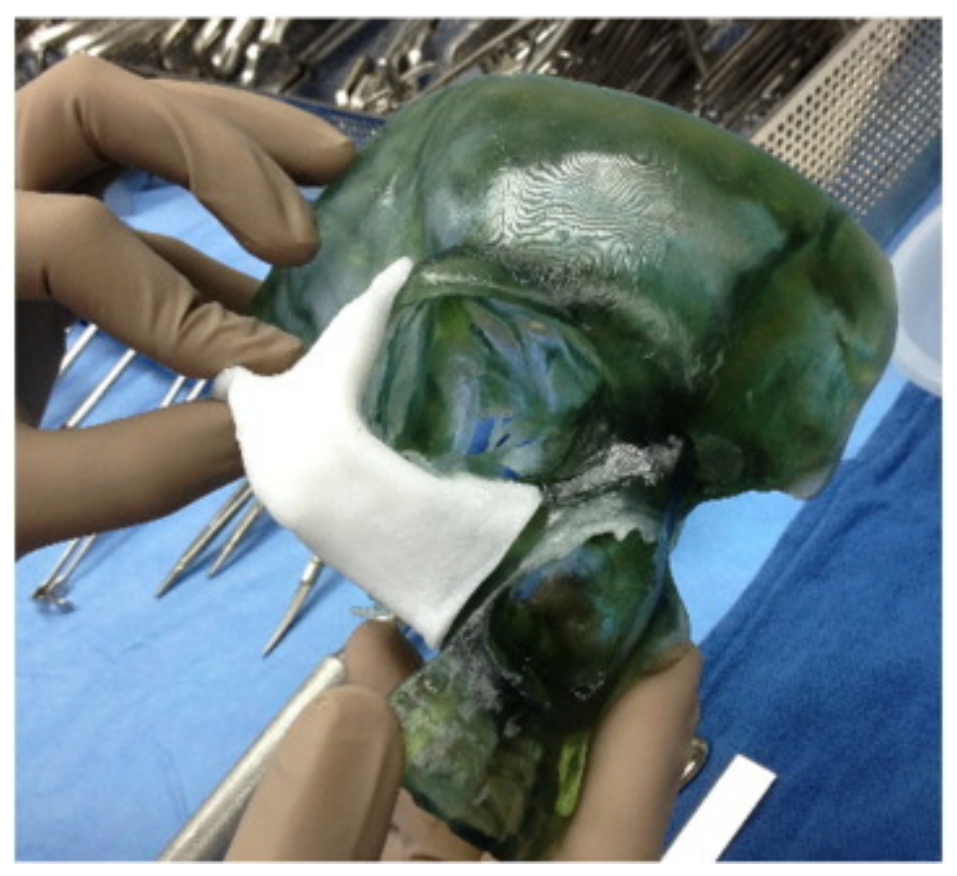

